\title{
Research in the Field of Prevention in Latin America
}

\section{Augusto Pérez-Gómez ${ }^{1}$ (D) Juliana Mejía-Trujillo ${ }^{1}$}

Published online: 9 March 2020

(C) Springer Science+Business Media, LLC, part of Springer Nature 2020

In the last decades, we have observed important changes in the field of alcohol and drug abuse prevention. At the request of the Editor-in-Chief of the International Journal of Mental Health and Addiction, we have assembled a series of papers examining the current state of addiction in several countries, particularly in South and Latin America.

Systematic research on addiction is not very common in Latin America; in recent years, many efforts have been undertaken to address the need to establish international standards before implementing massive and often costly intervention strategies. This Special Issue highlights some of these initiatives. Many countries in Latin America might be inspired by the examples presented in this special issue:

Menezes and colleagues evaluated user engagement and sustainability in the Strengthening Families Program (SFP), a prevention program in Brazil, by using semi-structured interviews with the facilitators.

Castaño and colleagues examined the adapted version of SFP and demonstrated that the intervention improved the quality of parenting, but no changes were observed regarding risk behaviors in adolescents.

Mejía and colleagues from Panama presented a critical review of existing family skills training programs in several Latin American countries, and suggested a series of strategies to improve those programs.

Reyes and colleagues presented a randomized control trial aimed at evaluating the efficacy of a prevention program developed and implemented in Colombia, based on motivational interviewing. The results are very encouraging, showing a decrease of alcohol use in adolescents aged 14-15, from 30 to $15 \%$. This program is also currently implemented in Mexico and Brazil.

Millé and colleagues in Mexico evaluated a prevention program titled Creciendo Juntos (Growing Up Together). The impact evaluation demonstrated favorable modification of drug use, showing a decrease of more than $30 \%$ in tobacco use in the past year and problems associated with alcohol use in all age groups, as well as a decrease of $25 \%$ in marijuana use in the past year.

Augusto Pérez-Gómez

aperez@nuevosrumbos.org

1 Corporación Nuevos Rumbos, Bogotá, D.C., Colombia 
Alonso and Romero examined the relationship between problematic internet use (PIU) and personality variables, such as neuroticism, extraversion, agreeableness, openness, and conscientiousness. The results suggest that different personal mechanisms may be involved in several dimensions constituting PIU in Spain.

Redi-Lago and colleagues explored the concept of "relational autonomy" of people with substance use disorders who used mental health services in Brazil.

Pérez-Gómez and colleagues examined the relationship between drug abuse and 13 categories of health problems in Colombia. They identified three main sets of health disorders: respiratory, psychological, and digestive.

Salazar and colleagues in Cuba compared patients with alcohol dependence (AD) without other psychiatric conditions and patients with dual diagnosis (AD plus another psychiatric problem) regarding the possibility of greater neurocognitive damage. Their results showed that patients with dual diagnosis showed more neurocognitive deficits and a more severe pattern of alcohol use.

Ruybal and Crano examined the influence of the parent-children relationship on occurrence of marijuana use, using data from the 2014 National Survey on Drug Use and Health (NSDUH) of the USA. They investigated the hypothesis that lower levels of parental monitoring and warmth are inversely associated with adolescent depressive symptoms and marijuana use. Their results confirmed their hypothesis.

Noyola and colleagues carried out a qualitative analysis aimed at understanding the pros and cons of continuing to use drugs among users with comorbid depressive symptoms in Mexico City. They identified nine themes, the most common being "mental health and emotional well-being" and "beliefs and expectations about substances." The least common themes were "family relationships," "sexuality," and "functioning in the workplace."

De Oliveira Correa and colleagues evaluated a community's readiness to implement interventions in Colombia. Findings suggest that community readiness can be measured adequately as a latent construct, and that its indicators can be used diagnostically to assess areas where readiness could be improved for better implementation of evidence-based interventions.

Valdes and colleagues presented the construction and validation of a diagnostic questionnaire aimed at detecting awareness of the consequences of using drugs in Chile; the authors found that levels of awareness increased significantly after 6 and 12 months of treatment.

Cabanillas from Peru examined epidemiological data to identify predictors of marijuana use in the general population. The findings suggest the need to consider gender-specific risk factors in the design and implementation of preventive programs for marijuana use.

Moreta-Herrera and colleagues examined patterns of alcohol consumption, intervention requirements, and sex differences in alcohol use within a sample of teenagers from Salcedo city, Ecuador.

Toledo-Fernández and colleagues from Mexico evaluated the differential effects of "cognitive reserve" of neurocognitive variables and determined which variables of polysubstance use predicted class membership.

Hernández-Llanes and colleagues employed a controlled Interrupted Time Series Analysis (ITSA) model in order to assess the effect of enforcing the ban of alcohol sales to underage individuals at the national level in Mexico.

Conde and colleagues from Argentina presented a mixed-methods study identifying reasons for delaying help-seeking, as well as the time gap between drinking initiation and problem 
acknowledgement. The authors argue that their findings would be useful for designing interventions aimed at reducing help-seeking barriers and improving access to treatment.

Augusto Pérez-Gómez, Ph.-D. and Juliana Mejía-Trujillo, M. A.

Guest Editors

Publisher's Note Springer Nature remains neutral with regard to jurisdictional claims in published maps and institutional affiliations. 